\title{
$\beta$-amyloid deposits in veins in patients with cerebral amyloid angiopathy and intracerebral haemorrhage
}

\author{
Tadeusz Mendel', Teresa Wierzba-Bobrowicz², Tomasz Stępień², Grażyna Maria Szpak² \\ ${ }^{12}{ }^{\text {nd }}$ Department of Neurology, ${ }^{2}$ Department of Neuropathology, Institute of Psychiatry and Neurology, Warsaw, Poland
}

\begin{abstract}
Aim of the study: To review the incidence and grade of cerebral amyloid angiopathy (CAA) in veins in patients who died due to spontaneous intracerebral haemorrhage (ICH).

Material and methods: Neuropathological examinations were performed in the study group of 189 patients. Cerebral amyloid angiopathy was diagnosed according to the Boston criteria and confirmed during an autopsy. The Vonsattel and Mountjoy scales were used to assess the grade and scores of CAA.

Results: In the study group composed of 189 ICH patients, 42 presented CAA. In the microscopic examination, of the 42 patients 33 (78\%) showed $\beta$-amyloid deposits in veins, which makes 17\% of the total group of patients with ICH. In this group, the age ranged from 54 to 97 (mean age $80.18 \pm 8.15$ years). A group of 33 (27 women and 6 men) patients comprised 15 (45\%) patients with severe CAA, 13 (40\%) with moderate and 5 (15\%) with mild CAA classified according to the Vonsattel scale. According to the Mountjoy scale 28 (85\%) patients had a score of 4, which indicated the total involvement of the vessel.

Conclusions: $\beta$-amyloid deposits in veins were found in $78 \%$ of patients with CAA and ICH, which makes $17 \%$ of the total group of patients with ICH. Interestingly, $\beta$-amyloid deposits in veins are not so rare in patients with CAA who died due to intracerebral haemorrhage. Cerebral amyloid angiopathy localization in the veins of the brain was observed more frequently than previously suspected. Veins may play a role in the elimination of $\beta$-amyloid from the brain.
\end{abstract}

Key words: cerebral amyloid angiopathy (CAA), $\beta$-amyloid, veins, intracerebral haemorrhage (ICH).

\section{Introduction}

Cerebral amyloid angiopathy (CAA), also known as congophilic amyloid angiopathy, is a progressive degenerative disease in which protein $\beta$-amyloid is deposited in the leptomeningeal and brain cortical small- and middle-sized arteries, veins and capillary walls. Cerebral amyloid angiopathy is the second cause of brain haemorrhage after atherosclerosis. It can also cause ischemic strokes, transient ischemic attacks, leukoencephalopathy, brain tumour (amyloidoma) and inflammatory vasculitis (A $\beta$-related angiitis). Cerebral amyloid angiopathy coexists with Alzheimer's disease (AD), dementia with Lewy bodies, Down syndrome and many other dementia-inducing disorders [1-4,7,13,17, $18,21,22]$. In cerebral amyloid angiopathy, intracerebral haemorrhages $(\mathrm{ICH})$ are superficial, multiple and occur in older people mostly with no history of hypertension $[7,16]$. The incidence of spontaneous cerebral amyloid angiopathy becomes more common with the increasing age; from $2.3 \%$ to $22 \%$ at the age of 65 and 
from $70 \%$ to $100 \%$ over the age of 80 years with a prevalence in AD (78-100\%) [12]. The prevalence of CAA in intracerebral haemorrhages ranged from $2.0 \%$ to $23 \%$ [12]; $30-40$ cases of CAA per 100,000 population have been recorded annually [7].

Cerebral veins are rarely involved in $\beta$-amyloid [25]. There are no publications concerning the involvement of veins in CAA in ICH. The aim of the study was to assess the incidence and grade of CAA in the veins of patients who died due to spontaneous ICH.

\section{Material and methods}

All patients were hospitalized in the $2^{\text {nd }}$ Department of Neurology, Institute of Psychiatry and Neurology, Warsaw, Poland. The study group consisted of patients who died due to spontaneous intracerebral haemorrhages in 1993-2012. The final diagnosis was made during an autopsy following histological and immunohistological examinations according to the Boston criteria [15].

Patients with suspected ICH were admitted to our hospital. They were hospitalized in the Stroke Unit. Over 20 years 189 patients died due to intracerebral haemorrhage of whom 42 were postmortem diagnosed with CAA. CAA was confirmed during a neuropathological examination. In 33 cases, veins were affected by CAA. Patients with brain aneurysm, arteriovenous malformation, brain tumour, venous thrombosis, haemophilias, past head injury, as well as those on anticoagulants and thrombolytic therapy were excluded.

The autopsy was performed according to the standard protocol. The brain was fixed in $4 \%$ paraformaldehyde in $0.1 \mathrm{M}$ phosphorane-buffer saline and embedded in paraffin. Then, it was sliced coronally. The specimens were stained with H\&E, PAS, Congo red and immunohistochemically with the two antibodies: anti A $\beta$ 8-17 (DAKO, $1: 50$ ) and actin (SMA, DAKO, $1: 50$ ).

Under the microscopic evaluation the brain was considered positive for CAA when it showed at least one leptomeningeal or cortical congophilic vessel with yellow-green birefringence under polarized light and/or $\beta$-amyloid deposits were seen within the wall of the vessels. The severity of CAA was classified according to the Vonsattel scale (Fig. 1):

1) mild - deposits of $\beta$-amyloid are restricted to a congophilic rim around normal or atrophic smooth muscle fibers (which might be absent), leaving an optically empty tiny vacuole-like zone surrounded by amyloid, in media of otherwise normal vessels,

2 ) moderate - media is replaced by $\beta$-amyloid and thicker than normal, with no evidence of remote or recent blood leakage,
3) severe - there are extensive $\beta$-amyloid deposits with focal wall fragmentation and at least one focus of perivascular leakage evidenced by the presence of erythrocytes or hemosiderin or both [24].

The estimated proportion of $\beta$-amyloid involvement in each vessel was recorded according to the Mountjoy scale (Fig. 2). A score of 0 indicated the absence of amyloid; a score of 1 - involvement of up to onequarter of the vessel circumference; a score of 2 involvement of up to one-half of the vessel circumference; a score of 3 -involvement of up to three-quarters of the vessel circumference; and a score of 4 indicated total involvement of the vessel [19].

\section{Results}

The results of the study focused on the clinical and neuropathological findings in cerebral amyloid angiopathy in patients who died due to spontaneous intracerebral haemorrhages are presented.

Deposits of $\beta$-amyloid CAA were observed in all kinds of cerebral vessels, arteries (cortical and meningeal), capillaries and veins. The neuropathological features localized in veins are presented in Table I.

In the group of 189 patients with $\mathrm{ICH}$, we found 42 patients with CAA. In the CAA group, we revealed 33 (78\%) patients with $\beta$-amyloid deposits in veins, which made $17 \%$ of the total group of patients who died due to $\mathrm{ICH}$. In this group, there were 27 women and 6 men; their age ranged from 54 to 97 (mean age $80.18 \pm 8.15$ years)

The grade of CAA according to the Vonsattel scale is presented in Table II. The following grades were identified: severe CAA in 15 (45\%) patients, moderate in 13 (40\%) and mild CAA in 5 (15\%) patients. According to the Mountjoy scale, we found score 4 in 28 (85\%) patients, score 3 in $3(9 \%)$, score 2 in 1 (3\%) and score 1 in 1 (3\%) patient (Table III).

We found one patient with Down syndrome and $\mathrm{ICH}$ who had veins involved with severe CAA and one patient suffering from dementia with Lewy bodies and ICH who had veins involved with moderate CAA.

\section{Discussion}

To our best knowledge, there are no reports on the veins involvement in $\mathrm{CAA}$ or $\mathrm{ICH}$. Therefore, we tried to find out how often the brain veins are deposited by $\beta$-amyloid.

Neuropathological examination is still a "gold standard" for CAA diagnosis. Using the Boston criteria for 

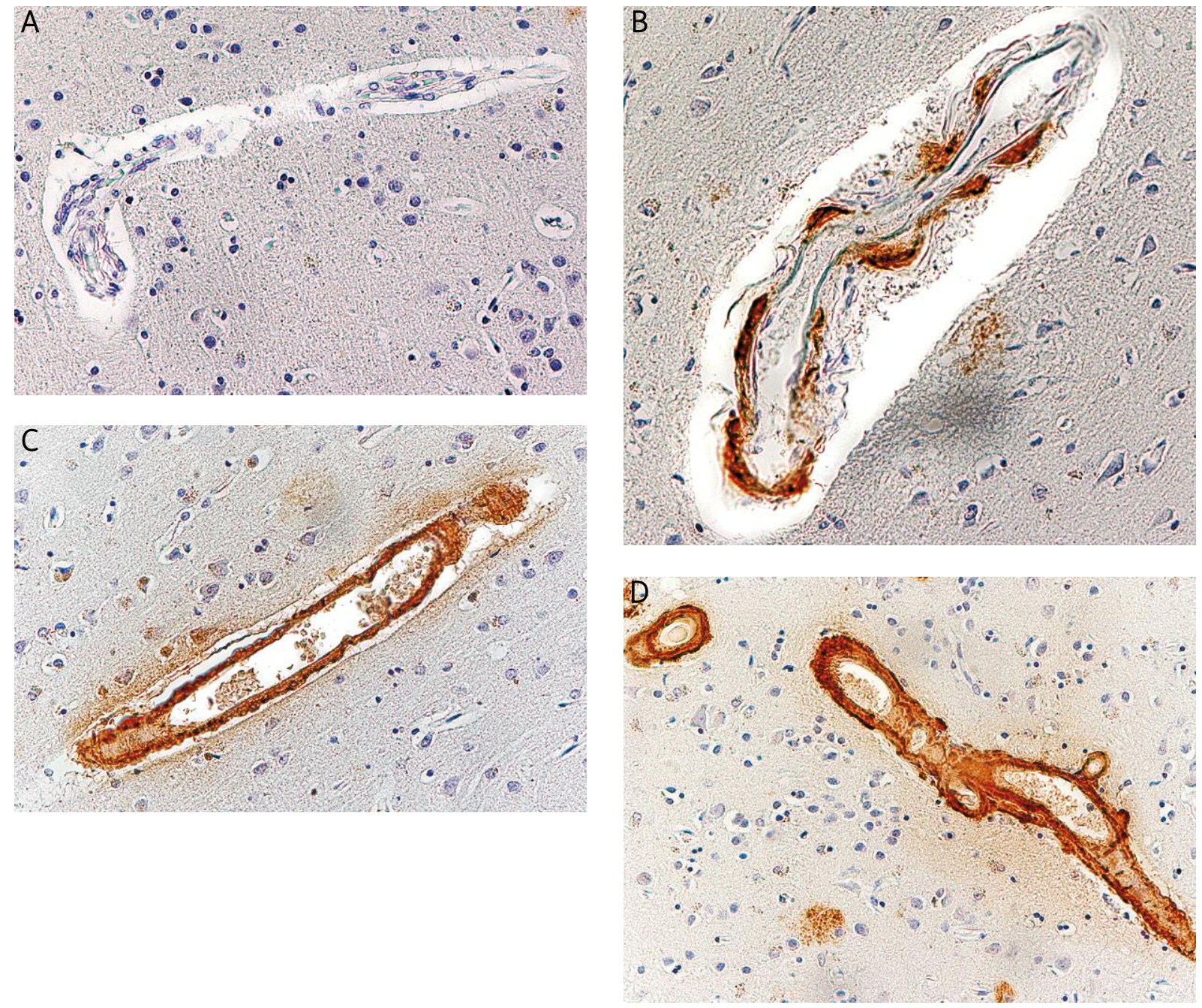

Fig. 1. The severity of CAA according to the Vonsattel scale was classified as follows: A) normal vein, without deposits of $\beta$-amyloid. $\beta$-amyloid. x 200; B) 1 . Mild - $\beta$-amyloid is restricted to segmental $\beta$-amyloid depositions. $\beta$-amyloid. $\times 200 ; C) 2$. Moderate - Media is replaced by $\beta$-amyloid and is thicker than normal, with no evidence of remote or recent blood leakage. $\beta$-amyloid. $x$ 200; D) 3. Severe - there is extensive amyloid deposition with focal wall fragmentation and focus of perivascular leakage. $\beta$-amyloid. $x 200$.

CAA diagnosis and having CT and/or MRI scans the neurologist may select those of the patients whom he or she most suspects of having CAA $[6,8,9,11,14,15,20,23]$. Also positron emission tomography (PET) scans can be used in the diagnosis of $\beta$-amyloid deposition in the brain [5].

A definite diagnosis of CAA can be made through a postmortem examination or obtained via intracranial biopsy, while on the basis of clinical data, and CT or MRI scans of the brain, CAA can be only suspected $[7,10,15,19,24]$.
There is a lot of diagnostic tools which can be used in the diagnosis of CAA during intracerebral haemorrhage, but only neuropathological examination can confirm the presence of this disease and reveal CAA stages, as well as the kinds of vessels involved in this process. It can allow to classify the grades and scores of CAA and its localization $[7,10,15,19,24]$.

There are only a few ways to eliminate $\beta$-amyloid from the brain. Imbalance between $\beta$-amyloid production and clearance is generally considered a key element in the formation of amyloid deposits. The amphiphilic 

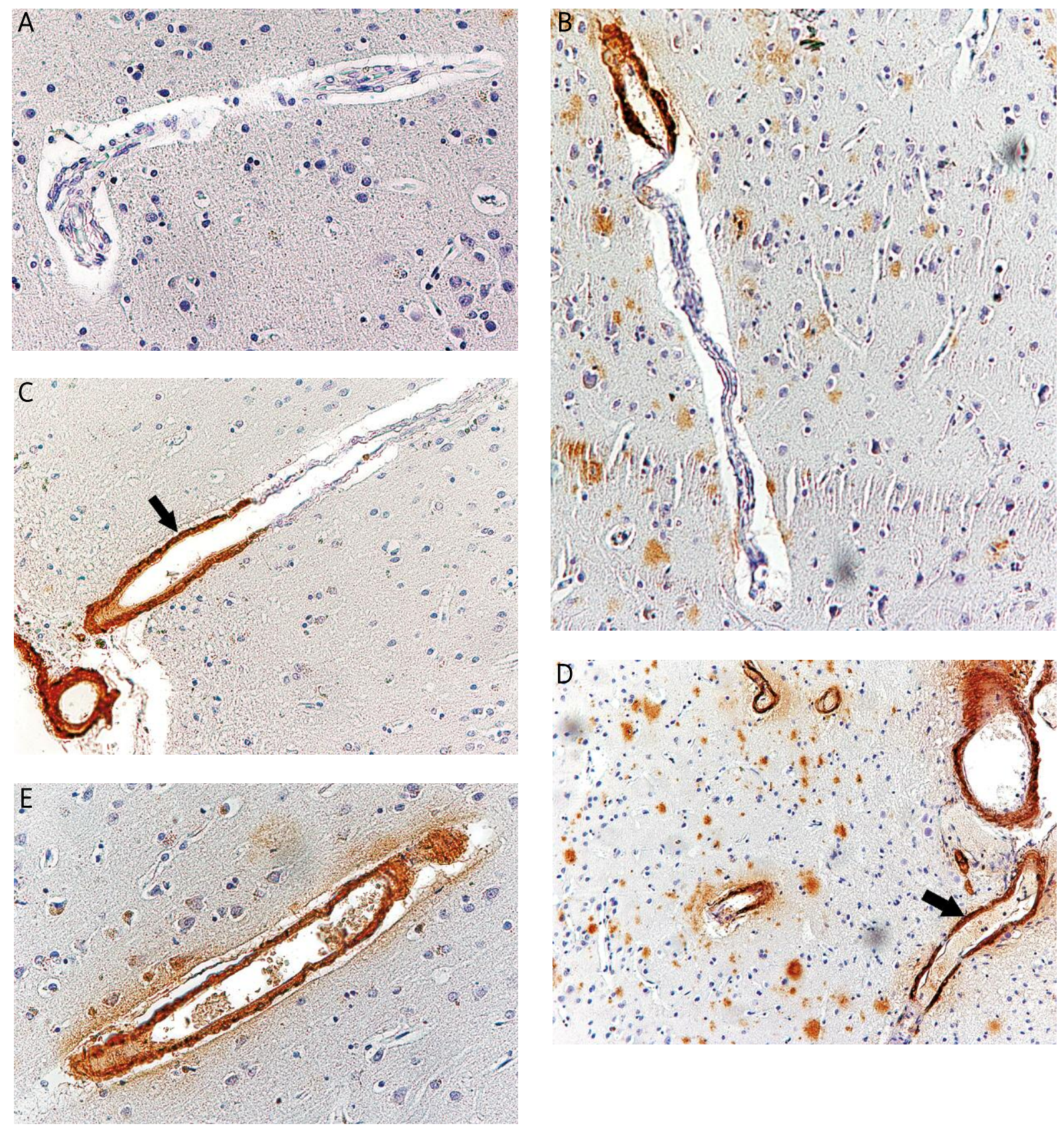

Fig. 2. The estimated proportion of amyloid involvement in each vessel was recorded according to the Mountjoy scale. A) A score of 0 indicated the absence of $\beta$-amyloid deposits. $\beta$-amyloid. $\times 200$; B) A score of 1 - involvement of up to one-quarter of the vessel circumference. $\beta$-amyloid. $x 100 ; C$ ) A score of 2 - involvement of up to one-half of the vessel circumference (arrow). $\beta$-amyloid. x 200; D) A score of 3 -involvement of up to threequarters of the vessel circumference (arrow). $\beta$-amyloid. $\times 100$; E) A score of 4 - total involvement of the vessel. $\beta$-amyloid. $\times 200$.

nature of $\beta$-amyloid precludes its crossing through the blood-brain barrier unless mediated by specialized carriers and/or receptor transport mechanisms. These mechanisms control the uptake of circulating $\beta$-amy- loid into the brain and regulate clearance. Among receptors involved, receptor for Advance Glycation End-products actively participates in brain uptake of free $\beta$-amyloid at the vessel wall level. Other receptors are more 
Table I. Cerebral amyloid angiopathy severity in veins of patients with intracerebral haemorrhages

\begin{tabular}{|c|c|c|c|c|}
\hline Number/Initials & Age & Sex & $\begin{array}{l}\text { CAA severity according } \\
\text { to the Vonsattel scale }\end{array}$ & $\begin{array}{l}\text { CAA severity according } \\
\text { to the Mountjoy scale }\end{array}$ \\
\hline $1 / W P$ & 84 & $\mathrm{~F}$ & 2 & 4 \\
\hline $2 / W P$ & 85 & $\mathrm{~F}$ & 2 & 4 \\
\hline $3 / R Z$ & 87 & $\mathrm{~F}$ & 1 & 3 \\
\hline $4 / A P$ & 83 & $\mathrm{~F}$ & 3 & 4 \\
\hline $5 / K T$ & 97 & $\mathrm{~F}$ & 2 & 4 \\
\hline 6/BP & 68 & $\mathrm{~F}$ & 2 & 4 \\
\hline $7 / \mathrm{HW}$ & 72 & $\mathrm{~F}$ & 1 & 3 \\
\hline 8/TN & 89 & $M$ & 3 & 4 \\
\hline 9/DK & 80 & $\mathrm{~F}$ & 2 & 4 \\
\hline $10 / S P$ & 68 & $M$ & 2 & 4 \\
\hline 11/ZO & 89 & $\mathrm{~F}$ & 2 & 4 \\
\hline $12 / R B$ & 77 & $\mathrm{~F}$ & 3 & 4 \\
\hline $13 / \mathrm{SD}$ & 84 & $M$ & 1 & 2 \\
\hline $14 / A P$ & 87 & $\mathrm{~F}$ & 3 & 4 \\
\hline 15/AWZ & 72 & $\mathrm{~F}$ & 3 & 4 \\
\hline 16/EC & 89 & M & 2 & 4 \\
\hline $17 / P W$ & 54 & $\mathrm{~F}$ & 3 & 4 \\
\hline $18 / \mathrm{SO}$ & 78 & $\mathrm{~F}$ & 2 & 4 \\
\hline 19/JZ & 85 & M & 3 & 4 \\
\hline 20/MK & 82 & $\mathrm{~F}$ & 3 & 4 \\
\hline $21 / A O$ & 77 & $\mathrm{~F}$ & 3 & 4 \\
\hline $22 / R S$ & 79 & $\mathrm{~F}$ & 3 & 4 \\
\hline 23/MSG & 79 & $\mathrm{~F}$ & 3 & 4 \\
\hline $24 / J F$ & 81 & $\mathrm{~F}$ & 3 & 4 \\
\hline 25/HK & 79 & $\mathrm{~F}$ & 1 & 3 \\
\hline 26/MM & 75 & $\mathrm{~F}$ & 3 & 4 \\
\hline 27/EK & 67 & $\mathrm{~F}$ & 2 & 4 \\
\hline $28 / S Z$ & 91 & $\mathrm{~F}$ & 3 & 4 \\
\hline 29/JP & 88 & F & 2 & 4 \\
\hline $30 / \mathrm{HG}$ & 71 & $M$ & 2 & 4 \\
\hline $31 / A R$ & 84 & $\mathrm{~F}$ & 1 & 1 \\
\hline $32 / K M$ & 77 & $\mathrm{~F}$ & 3 & 4 \\
\hline 33/WR & 88 & $\mathrm{~F}$ & 2 & 4 \\
\hline
\end{tabular}


Table II. Cerebral amyloid angiopathy grades according to the Vonsattel scale

\begin{tabular}{|lc|}
\hline CAA grade & Number $(\%)$ \\
\hline Mild & $15(45 \%)$ \\
\hline Moderate & $13(40 \%)$ \\
\hline Severe & $5(15 \%)$ \\
\hline
\end{tabular}

relevant to the transport of $\beta$-amyloid complexed with other molecules: low-density-lipoprotein receptor related protein-1 (LRP-1) mediated transcytosis of $\beta$-amyloid-ApoE complexes contributing to rapid CNS clearance, whereas megalin mediates the cellular uptake and transport of $\beta$-amyloid-ApoJ. Neprilysin, endothelinamyloid-converting enzyme, insulin-degrading enzyme, beta-amyloid-converting enzyme 1, plasmin and matrix metalloproteases are among major enzymes known to participate in brain $\beta$-amyloid catabolic pathways. Reduced levels and/or activity of $\beta$-amyloid degrading enzymes favour $\beta$-amyloid accumulation causing increased levels of $\beta$-amyloid deposits. $\beta$-amyloid can be also eliminated from the brain by the perivascular drainage. There is no evidence that all these receptors are located in all kinds of cerebral vessels [3].

Brain veins localized in the grey and white matter have a relatively large lumen and thin walls that lack smooth muscle cells. This characteristic distinguishes veins from arteries. The leptomeningeal cells around veins do not form a complete sheath and there is a perivascular space around veins containing a few collagen fibers. Postcapillary venules are the site for the receptor-mediated entry of inflammatory cells into the brain [25].

According to Weller et al. (2009), veins do not appear to be involved in CAA, although deposits of $\beta$-amyloid are found attached to the walls of veins [25].

In our microscopic findings, we found veins involved in 33 (78\%) patients. According to both Vonsattel and Mountjoy scales, severe and moderate changes were dominating in our group of patients. Based on our results we may assume that veins may play a more important role in $\beta$-amyloid elimination from the brain than we previously thought.

\section{Conclusions}

We found $\beta$-amyloid deposited in veins in $78 \%$ of patients with CAA and ICH. Veins are not so rarely in-
Table III. Cerebral amyloid angiopathy scores according to the Mountjoy scale

\begin{tabular}{|cc|}
\hline CAA score & Number (\%) \\
\hline 1 & $1(3 \%)$ \\
\hline 2 & $1(3 \%)$ \\
\hline 3 & $3(9 \%)$ \\
\hline 4 & $28(85 \%)$ \\
\hline
\end{tabular}

volved in $\beta$-amyloid in intracerebral haemorrhage. Cerebral amyloid angiopathy was localized in brain veins more often than we previously suspected. Veins may play an important role in the elimination of $\beta$-amyloid and not only as a perivascular drainage.

\section{References}

1. Armstrong RA. $\beta$-amyloid (A $\beta$ ) deposition in cognitively normal brain, dementia with Lewy bodies, and Alzheimer's disease: a study using principal components analysis. Folia Neuropathol 2012; 50: 130-139.

2. Armstrong RA. Size frequency distributions of $\beta$-amyloid $(A \beta)$ deposits: a comparative study of four neurodegenerative disorders. Folia Neuropathol 2012; 50: 240-249.

3. Biffi A, Greenberg SM. Cerebral amyloid angiopathy: a systematic review. J Clin Neurol 2011; 7: 1-9.

4. Charidimou A, Gang Q, Werring DJ. Sporadic cerebral amyloid angiopathy revisited: recent insights into pathophysiology and clinical spectrum. J Neurol Neurosurg Psychiatry 2012; 83: 124-137.

5. Forsberg A, Engler H, Almkvist O, Blomquist G, Hagman G, Wall A, Ringheim A, Långstöm B, Nordberg A. PET imaging of amyloid deposition in patients with mild cognitive impairment. Neurobiol Aging 2008; 29: 1456-1465.

6. Ghostine S, Raghavan R, Khanlou N, Vinters HV, Tong KA, Johnson WD, Oyoyo U, Kido D. Cerebral amyloid angiopathy: microhaemorrhges demonstrated by magnetic resonance susceptibility-weighted imaging. Neuropathol App Neurobiol 2009; 35: 116-119.

7. Greenberg SM. Cerebral amyloid angiopathy: Prospects for clinical diagnosis and treatment. Neurology 1998; 51: 690-694.

8. Greenberg SM, O'Donnell HC, Schaefer PW, Kraft E. MRI detection of new hemorrhages: Potential marker of progression in cerebral amyloid angiopathy. Neurology 1999; 53: 1135-1138.

9. Haacke FM, DelProposto ZS, Chaturvedi S, Seghal V, Tenzer M, Neelavalli J, Kido D. Imaging cerebral amyloid angiopathy with susceptibility-weighted imaging. AJNR Am J Neuroradiol 2007; 28: 316-317.

10. Hendricks HT, Franke CL, Theunissen PHMH. Cerebral amyloid angiopathy: diagnosis by MRI and brain biopsy. Neurology 1990; 40: 1308-1310.

11. Imaizumi T, Honma T, Horita Y, Kawamura M, Kohama I, Miyata K, Nyon KS, Niwa J. The number of microbleeds on gradient $\mathrm{T}^{*}$ weighted magnetic resonance image at the onset of intracerebral hemorrhage. J Stroke Cerebrovasc Dis 2008; 17: 30-34. 
12. Jellinger KA, Lauda F, Attems J. Sporadic cerebral amyloid angiopathy is not a frequent cause of spontaneous brain hemorrhage. Eur J Neurol 2007; 14: 923-928.

13. Karbowniczek A, Wierzba-Bobrowicz T, Mendel T, Nauman P. Cerebral amyloid angiopathy manifestated as a brain tumour. Clinical and neuropathological characteristics of two cases. Folia Neuropathol 2012; 50: 194-200.

14. Kidwell CS, Wintermark M. Imaging of intracranial haemorrhage. Lancet Neurol 2008; 7: 256-267.

15. Knudsen KA, Rosand J, Karluk D, Greenberg SM. Clinical diagnosis of cerebral amyloid angiopathy: validation of the Boston Criteria. Neurology 2001; 56: 537-539.

16. Lopez JR, Fontanillo MMF, Carretero MJM, Garcia EC, Jaime DE, Montero JLM, Hermida MJP. Lobar intracerebral haemorrhage and cerebral amyloid angiopathy: analysis of a series of 106 patients. Neurologia 2009; 24: 386-390.

17. Mendel T, Bertrand E, Szpak GM, Stepień T, Wierzba-Bobrowicz T. Cerebral amyloid angiopathy as a cause of an extensive brain hemorrhage in adult patient with Down's syndrome - a case report. Folia Neuropathol 2010; 48: 206-211.

18. Mendel T, Bertrand E, Szpak GM, Stępień T, Wierzba-Bobrowicz T. Complications of severe cerebral amyloid angiopathy in the course of dementia with Lewy bodies. A case report. Folia Neuropathol 2010; 48: 293-299.

19. Mountjoy CQ, Tomlinson BE, Gibson PH. Amyloid and senile plaques and cerebral blood vessels. J Neurol Sci 1982; 57: 89-103.

20. Nandigam RNK, Viswanathan A, Delgado P, Skehan ME, Smith EE, Rosand J, Greenberg SM, Dickerson BC. MR imaging detection of cerebral microbleeds: Effect of susceptibility-weighted imaging, section thickness, and field strength. AJNR 2009; 30: 338-343.

21. Pluta R, Jabłoński M, Czuczwar SJ. Postischemic dementia with Alzheimer phenotype: selectively vulnerable versus resistant areas of the brain and neurodegeneration versus $\beta$-amyloid peptide. Folia Neuropathol 2012; 50: 101-109.

22. Szpak GM, Lewandowska E, Śliwińska A, Stępień T, Tarka S, Mendel T, Rafałowska J. Inflammatory cerebral amyloid angiopathy: the overlap of perivascular (PAN-like) with vasculitic (Aß-related angiitis) form: an autopsy case. Folia Neuropathol 2011; 49: 335-347.

23. Torii T, Nakamori M, Kubo S, Sugiura T, Yamada A. Cerebral amyloid angiopathy with varied hemorrhage pattern on T2*-weighted magnetic resonance imaging. Intern Med 2009; 48: 735-736.

24. Vonsattel JPG, Myers RH, Hedley-Whyte ET, Ropper AH, Bird ED, Richardson EP. Cerebral amyloid angiopathy without and with cerebral hemorrhages: A comparative histological study. Ann Neurol 1991; 30: 637-649.

25. Weller R, Boche D, Nicoll JAR. Microvasculature changes and cerebral amyloid angiopathy in Alzheimer's disease and their potential impact on therapy. Acta Neuropathol 2009; 118: 87-102. 